\title{
ON THE MAZUR-ULAM THEOREM AND THE ALEKSANDROV PROBLEM FOR UNIT DISTANCE PRESERVING MAPPINGS
}

\author{
THEMISTOCLES M. RASSIAS AND PETER ŠEMRL
}

(Communicated by Palle E. T. Jorgensen)

\begin{abstract}
Let $X$ and $Y$ be two real normed vector spaces. A mapping $f: X \longrightarrow Y$ preserves unit distance in both directions iff for all $x, y \in X$ with $\|x-y\|=1$ it follows that $\|f(x)-f(y)\|=1$ and conversely. In this paper we shall study, instead of isometries, mappings satisfying the weaker assumption that they preserve unit distance in both directions. We shall prove that such mappings are not very far from being isometries. This problem was asked by A. D. Aleksandrov. The first classical result that characterizes isometries between normed real vector spaces goes back to $S$. Mazur and S. Ulam in 1932. We also obtain an extension of the Mazur-Ulam theorem.
\end{abstract}

\section{INTRODUCTION}

Let $X$ and $Y$ be two real normed vector spaces. A mapping $f: X \rightarrow Y$, of $X$ onto $Y$, is defined to be an isometry if

$$
\|f(x)-f(y)\|=\|x-y\| \quad \text { for all } x, y \in X .
$$

If a mapping $f$ of $X$ onto $Y$ is an isometry then the inverse mapping $f^{-1}: Y$ $\longrightarrow X$ is an isometry of $Y$ onto $X$. Two normed spaces $X$ and $Y$ are defined to be isometric if there exists an isometry of $X$ onto $Y$. Mazur and Ulam [3] proved that every isometry of a normed real vector space onto a normed real vector space is a linear mapping up to translation. Consider then the following conditions for the mapping $f: X \longrightarrow Y$.

- A mapping $f: X \rightarrow Y$ satisfies the distance one preserving property (DOPP) iff for all $x, y \in X$ with $\|x-y\|=1$ it follows that $\|f(x)-f(y)\|=1$.

- A mapping $f: X \rightarrow Y$ satisfies the strong distance one preserving property (SDOPP) iff for all $x, y \in X$ with $\|x-y\|=1$ it follows that $\| f(x)-$ $f(y) \|=1$ and conversely.

Problem (P). Let $f: X \longrightarrow Y$ be a mapping (not necessarily continuous) satisfying condition (DOPP). Is $f: X \longrightarrow f(X) \subset Y$ an isometry? (cf. [5-7].)

It seems the study of Problem (P) was started by Aleksandrov [1]. Some results concerning this question are given in $[2,4]$ where some further references

Received by the editors March 26, 1991 and, in revised form, June 24, 1991.

1991 Mathematics Subject Classification. Primary 51K05.

The second author was partially supported by the Research Council of Slovenia. 
on the problem can be found. This problem still remains open even for the case where $X=\mathbb{R}^{n}$ and $Y=\mathbb{R}^{m}$ with $2 \leq n<m$. It is particularly interesting (and it seems to be a hard problem) to decide whether or not there is a unit distance preserving mapping $f: \mathbb{R}^{2} \longrightarrow \mathbb{R}^{3}$ that is not an isometry (cf. $[5,7]$ ).

\section{Main Results}

In this paper we shall study, instead of isometries, mappings satisfying the weaker assumption that they preserve unit distance in both directions. We shall see that such mappings are not very far from being isometries. More precisely, we shall prove

Theorem 1. Let $X$ and $Y$ be real normed vector spaces such that one of them has dimension greater than one. Suppose that $f: X \longrightarrow Y$ is a surjective mapping satisfying (SDOPP). Then $f$ is an injective mapping satisfying

$$
|\|f(x)-f(y)\|-\|x-y\||<1 \text { for all } x, y \in X .
$$

Moreover, $f$ preserves distance $n$ in both directions for any positive integer $n$. Proof. We shall show that both spaces have dimension greater than one. Let us first assume that $\operatorname{dim} Y \geq 2$. It follows that there exist vectors $x, y, z \in Y$ such that

$$
\|x-y\|=\|x-z\|=\|y-z\|=1 .
$$

The mapping $f$ is given to be surjective and to preserve distance one in both directions. Thus we can find $x_{1}, y_{1}, z_{1} \in X$ satisfying

$$
\left\|x_{1}-y_{1}\right\|=\left\|x_{1}-z_{1}\right\|=\left\|y_{1}-z_{1}\right\|=1 .
$$

This implies that $\operatorname{dim} X \geq 2$. Similarly, one can prove that if $\operatorname{dim} X \geq 2$ then $\operatorname{dim} Y \geq 2$. Claim that $f$ is injective. Suppose on the contrary, that there are $x, y \in X, x \neq y$, such that $f(x)=f(y)$. We can find a vector $z \in X$ such that $\|x-z\|=1$ and $\|z-y\| \neq 1$. Then $\|f(x)-f(z)\|=\|f(y)-f(z)\|=1$. This implies that $\|y-z\|=1$, which is a contradiction. Therefore $f$ is a bijective mapping. Both $f$ and $f^{-1}$ preserve unit distance.

In the sequel we shall need the following notations:

$$
\begin{aligned}
\bar{K}(x, r) & =\{z:\|z-x\| \leq r\}, \\
K(x, r) & =\{z:\|z-x\|<r\}, \\
C_{x}(n, n+1] & =\{z: n<\|z-x\| \leq n+1\} .
\end{aligned}
$$

Let $x$ be an arbitrary vector in $X$ and $n$ any positive integer, $n>1$. Assume that $z \in \bar{K}(x, n)$. Since $\operatorname{dim} X>1$, we can find a sequence $x=$ $x_{0}, x_{1}, \ldots, x_{n}=z$, such that

$$
\left\|x_{i+1}-x_{i}\right\|=1, \quad i=0,1,2, \ldots, n-1 .
$$

Consequently, we have $\left\|f\left(x_{n}\right)-f\left(x_{0}\right)\right\|=\|f(z)-f(x)\| \leq n$. Therefore,

$$
f(\bar{K}(x, n)) \subset \bar{K}(f(x), n) .
$$

The same result can be obtained for $f^{-1}$. Hence,

$$
f(\bar{K}(x, n))=\bar{K}(f(x), n), \quad x \in X, n \in \mathbb{N} \backslash\{1\} .
$$


However, $f$ is bijective and thus

$$
f\left(C_{x}(n, n+1]\right)=C_{f(x)}(n, n+1], \quad x \in X, n \in \mathbb{N} \backslash\{1\} .
$$

We fix an element $x \in X$ and choose $z \in C_{x}(1,2]$. We know that $f(z) \in$ $\bar{K}(f(x), 2)$. The vector $u=z+(\|z-x\|)^{-1}(z-x)$ is contained in $C_{x}(2,3]$. According to $(2)$ we have $f(u) \in C_{f(x)}(2,3]$. Thus,

$$
\|f(x)-f(u)\|>2 \text {. }
$$

Let us assume that $\|f(z)-f(x)\| \leq 1$ holds. Then we obtain

$$
\|f(x)-f(u)\| \leq\|f(x)-f(z)\|+\|f(z)-f(u)\|=\|f(x)-f(z)\|+1 \leq 2,
$$

which contradicts $(3)$. We have proved that

$$
f\left(C_{x}(1,2]\right) \subset C_{f(x)}(1,2] .
$$

The same result holds for the mapping $f^{-1}$. Consequently, the relations

$$
f\left(C_{x}(1,2]\right)=C_{f(x)}(1,2] \text { and } f(K(x, 1))=K(f(x), 1)
$$

hold for all $x \in X$. This together with (2) implies the inequality (1). In order to complete the proof we shall show by induction on $n$ that $f$ preserves distance $n$ in both directions for all positive integers $n$. Assume that $f$ preserves distance $n$ in both directions. Let $x$ and $z$ be vectors in $X$ such that $\|x-z\|=n+1$. It follows that $\|f(x)-f(z)\| \leq n+1$. Let us define $u$ by

$$
u=f(x)+\frac{f(z)-f(x)}{\|f(z)-f(x)\|} .
$$

Since $f$ is surjective we can find $v \in X$ such that $u=f(v)$. From $\|u-f(x)\|=$ 1 we obtain $\|v-x\|=1$. If $\|u-f(z)\|<n$ we get $\|v-z\|<n$. This together with $\|v-x\|=1$ further implies that $\|x-z\|<n+1$, which is a contradiction. Thus, we have necessarily that $\|u-f(z)\| \geq n$. This implies

$$
\begin{aligned}
n & \leq\|u-f(z)\|=\left\|f(x)\left(1-\frac{1}{\|f(x)-f(z)\|}\right)-f(z)\left(1-\frac{1}{\|f(x)-f(z)\|}\right)\right\| \\
& =|\|f(x)-f(z)\|-1| .
\end{aligned}
$$

Therefore, $\|f(x)-f(z)\|=n+1$. The same proof shows that $f^{-1}$ preserves distance $n+1$ as well. Q.E.D.

Proposition 2. The assumption that one of the spaces has dimension greater than one cannot be omitted in Theorem 1.

Proof. Let $f: \mathbb{R} \longrightarrow \mathbb{R}$ be defined by

$$
f(x)= \begin{cases}x+2 & \text { if } x \text { is an integer } \\ x & \text { if } x \text { is not an integer. }\end{cases}
$$

The mapping $f$ is bijective and preserves distance $n$ in both directions for any $n \in \mathbb{N}$. However, inequality (1) is not fulfilled. Q.E.D.

Proposition 3. In Theorem 1 (SDOPP) cannot be replaced by (DOPP).

Proof. Let $g:[0,1) \longrightarrow[0,1) \times \mathbb{R}$ be a bijective mapping and define $f: \mathbb{R} \longrightarrow$ $\mathbb{R}^{2}$ by

$$
f(t)=g(t-[t])+([t], 0),
$$


where $[t]$ denotes the integer part of $t$. Obviously, $f$ is a bijective mapping preserving distance $n$ for any $n \in \mathbb{N}$. However, (1) is not fulfilled. Q.E.D.

Proposition 4. The inequality (1) in Theorem 1 is sharp.

Proof. Choose $\varepsilon \in\left(0, \frac{1}{2}\right)$ and define $g_{\varepsilon}:[0,1] \rightarrow[0,1]$ by

$$
g_{\varepsilon}(t)= \begin{cases}\frac{\varepsilon}{1-\varepsilon} t & \text { if } t \in[0,1-\varepsilon], \\ \frac{1-\varepsilon}{\varepsilon} t+\left(2-\frac{1}{\varepsilon}\right) & \text { if } t \in[1-\varepsilon, 1] .\end{cases}
$$

Also define $h_{\varepsilon}: \mathbb{R} \longrightarrow \mathbb{R}$ by $h_{\varepsilon}(s)=[s]+g_{\varepsilon}(s-[s])$. Obviously, $h_{\varepsilon}$ is a monotonically increasing function satisfying

$$
\begin{array}{ll}
|s-t|=n \text { if and only if }\left|h_{\varepsilon}(s)-h_{\varepsilon}(t)\right|=n, & n=1,2,3, \ldots ; \\
|s-t| \leq n \text { if and only if }\left|h_{\varepsilon}(s)-h_{\varepsilon}(t)\right| \leq n, \quad n=1,2,3, \ldots ;
\end{array}
$$

and

$$
\left|h_{\varepsilon}(s)-h_{\varepsilon}(t)\right| \leq \frac{1-\varepsilon}{\varepsilon}|s-t| .
$$

Let $C[0,1]$ be the set of all real-valued continuous functions defined on $[0,1]$. We define for $x \in C[0,1]$ the norm $\|x\|=\max _{t \in[0,1]}|x(t)|$. Define $\phi_{\varepsilon}: C[0,1]$ $\longrightarrow C[0,1]$ by $\left(\phi_{\varepsilon}(x)\right)(t)=h_{\varepsilon}(x(t))$. Clearly, $\phi_{\varepsilon}$ is a bijective mapping, $\left(\phi_{\varepsilon}^{-1}(x)\right)(t)=h_{\varepsilon}^{-1}(x(t))$. Moreover, we have for an arbitrary pair $x, y \in$ $C[0,1]$ the following property: $\|x-y\|=n$ if and only if there exists $t_{0} \in$ $[0,1]$ such that $\left|x\left(t_{0}\right)-y\left(t_{0}\right)\right|=n$ and $|x(t)-y(t)| \leq n$ for all $t \in[0,1]$, and this holds if and only if $\left|h_{\varepsilon}\left(x\left(t_{0}\right)\right)-h_{\varepsilon}\left(y\left(t_{0}\right)\right)\right|=n$ and $\left|h_{\varepsilon}(x(t))-h_{\varepsilon}(y(t))\right| \leq n$ for $t \in[0,1]$, which is equivalent to $\left\|\phi_{\varepsilon}(x)-\phi_{\varepsilon}(y)\right\|=n$. Thus, $\phi_{\varepsilon}$ preserves distance $n$ in both directions for any positive integer $n$. Choose $x(t) \equiv$ $1-\varepsilon \in C[0,1], y(t) \equiv 1 \in C[0,1]$. Then $\|x-y\|=\varepsilon$. It is clear that $\left(\phi_{\varepsilon}(x)\right)(t) \equiv \varepsilon,\left(\phi_{\varepsilon}(y)\right)(t) \equiv 1$. Thus $\left\|\phi_{\varepsilon}(x)-\phi_{\varepsilon}(y)\right\|=1-\varepsilon$. Consequently,

$$
\left|\left\|\phi_{\varepsilon}(x)-\phi_{\varepsilon}(y)\right\|-\|x-y\|\right|=1-2 \varepsilon,
$$

and since $\varepsilon$ can be arbitrarily small, inequality (1) in Theorem (1) is sharp. Q.E.D.

Remark. The mapping $\phi_{\varepsilon}$ in Proposition 4 is not only continuous, but it satisfies a stronger condition (Lipschitz condition):

$$
\left\|\phi_{\varepsilon}(x)-\phi_{\varepsilon}(y)\right\| \leq K\|x-y\| \text { for } x, y \in X .
$$

This condition follows from (6) when $K=(1-\varepsilon) \varepsilon^{-1} \in(1, \infty)$. It follows that surjective Lipschitz mappings $(K>1)$ having (SDOPP) need not be isometries. However, in the case when $K=1$, we can prove an extension of Mazur-Ulam result.

Theorem 5. Let $X$ and $Y$ be real normed vector spaces such that one of them has dimension greater than one. Suppose that $f: X \longrightarrow Y$ is a Lipschitz mapping with $K=1$ :

$$
\|f(x)-f(y)\| \leq\|x-y\| \quad \text { for all } x, y \in X .
$$


Assume also that $f$ is a surjective mapping satisfying (SDOPP). Then $f$ is an isometry. Thus $f$ is a linear isometry up to translation.

Proof. According to Theorem 1, $f$ preserves distance $n$ in both directions for any positive integer $n$. Choose $x, y \in X$ and find a positive integer $n_{0}$ satisfying $\|x-y\|<n_{0}$. Assume that

$$
\|f(x)-f(y)\|<\|x-y\| .
$$

Set

$$
z=x+\frac{n_{0}}{\|y-x\|}(y-x) \text {. }
$$

Clearly,

$$
\|z-x\|=n_{0} \quad \text { and } \quad\|z-y\|=n_{0}-\|y-x\| .
$$

It follows that

$$
\|f(z)-f(x)\|=n_{0} \quad \text { and } \quad\|f(z)-f(y)\| \leq n_{0}-\|y-x\| .
$$

On the other hand,

$$
\begin{aligned}
\|f(z)-f(x)\| & \leq\|f(z)-f(y)\|+\|f(y)-f(x)\| \\
& <n_{0}-\|y-x\|+\|y-x\|=n_{0},
\end{aligned}
$$

which contradicts $\|f(z)-f(x)\|=n_{0}$. Therefore (7) is not valid. Hence

$$
\|f(x)-f(y)\|=\|x-y\| \quad \text { for all } x, y \in X,
$$

which implies that $f$ is an isometry. The Mazur-Ulam theorem now proves that $f$ is a linear mapping up to translation. Q.E.D.

Corollary 6. Let $X$ and $Y$ be real normed vector spaces such that one of them has dimension greater than one. Assume also that one of them is strictly convex. Suppose that $f: X \longrightarrow Y$ is a surjective mapping satisfying (SDOPP). Then $f$ is a linear isometry up to translation.

Remark. Let $X$ and $Y$ be real normed vector spaces such that $\operatorname{dim} X \geq 2$ and $Y$ is strictly convex. It is known (but to the best of our knowledge not yet published) that when $f: X \longrightarrow Y$ preserves two distances $a$ and $n a$ for a given $n \in \mathbb{N} \backslash\{1\}$ and a given $a \in \mathbb{R}^{+}, f$ is an isometry.

Proof. According to Theorem $1, f$ is a bijective mapping preserving distance $n$ in both directions for any positive integer $n$. Moreover, the proof of Theorem 1 shows that both spaces have dimension greater than one. So we can assume with no loss of generality that $Y$ is strictly convex.

We first prove that $f$ preserves distances $1 / n$ for every positive integer $n$. Choose $x, y \in X$ satisfying $\|x-y\|=1 / n$, and find a vector $z \in X$ such that $\|x-z\|=\|y-z\|=1$. Set

$$
u=z+n(y-z) \quad \text { and } \quad v=z+n(x-z) .
$$

Clearly, we have

$$
\|x-v\|=n-1 \quad \text { and }\|v-z\|=n
$$

It follows that

$$
\|f(x)-f(z)\|=1, \quad\|f(x)-f(v)\|=n-1, \quad\|f(v)-f(z)\|=n .
$$


As $Y$ is strictly convex, we have necessarily

$$
f(x)=\frac{1}{n} f(v)+\frac{n-1}{n} f(z)
$$

and, similarly,

$$
f(y)=\frac{1}{n} f(u)+\frac{n-1}{n} f(z) .
$$

Using $\|u-v\|=1$, we get $\|f(x)-f(y)\|=(1 / n)\|f(u)-f(v)\|=1 / n$.

Assume now that $\|x-y\| \leq(m / n)$, where $m \geq 2$ is a positive integer. As $\operatorname{dim} X>1$, we can find a finite sequence of vectors $x=z_{0}, z_{1}, \ldots, z_{m}=y$ such that $\left\|z_{i}-z_{i+1}\right\|=1 / n$. It follows that

$$
\|f(x)-f(y)\| \leq \sum_{i=0}^{m-1}\left\|f\left(z_{i}\right)-f\left(z_{i+1}\right)\right\|=\frac{m}{n} .
$$

Thus

$$
\|f(x)-f(y)\| \leq\|x-y\| \quad \text { for all } x, y \in X .
$$

The result follows now from Theorem 5. Q.E.D.

Corollary 7. Let $X$ and $Y$ be real normed vector spaces, $\operatorname{dim} X>1$, such that one of them is strictly convex. Suppose that $f: X \longrightarrow Y$ is a homeomorphism satisfying (DOPP). Then $f$ is a linear isometry up to translation.

Remark. This result was proved in [4] under the stronger assumption that $X=$ $Y=\mathbb{R}^{n},(3 \leq n \leq \infty)$.

Proof. For every vector $x \in X$ the unit sphere $S(x, 1)=\{y \in X:\|y-x\|=1\}$ is transformed into the sphere $S(f(x), 1)=\{y \in Y:\|y-f(x)\|=1\}$. Let $Z$ be the complement of $S(x, 1)$ in $X$ and $\bar{Z}$ the complement of $f(S(x, 1))$ in $Y$. While mapping $S(x, 1)$ onto $f(S(x, 1))$, the homeomorphism $f$ must simultaneously map $Z$ onto $\bar{Z}$. Claim that $f(S(x, 1))=S(f(x), 1)$. Suppose on the contrary that $f(S(x, 1))$ is a proper subset of $S(f(x), 1)$. Then its complement $\bar{Z}$ is connected, which is impossible since $\bar{Z}=f(Z)$ and $Z$ is disconnected. Thus, the unit sphere $S(x, 1)$ is transformed onto the unit sphere $S(f(x), 1)$. Consequently, the mapping $f$ satisfies (SDOPP). The result follows now from Corollary 6. Q.E.D.

\section{REFERENCES}

1. A. D. Alexandrov, Mappings of families of sets, Soviet Math. Dokl. 11 (1970), 116-120.

2. A. Guc, On mappings that preserve a family of sets in Hilbert and hyperbolic spaces, Candidate's Dessertation, Novosibirsk, 1973.

3. S. Mazur et S. Ulam, Sur les transformations isométriques d'espaces vectoriels normés, C. R. Acad. Sci. Paris 194 (1932), 946-948.

4. B. Mielnik and T. M. Rassias, On the Aleksandrov problem of conservative distances, Proc. Amer. Math. Soc. 116 (1992), 1115-1118.

5. T. M. Rassias, Is a distance one preserving mapping between metric spaces always an isometry?, Amer. Math. Monthly 90 (1983), 200. 
6. _. Some remarks on isometric mappings, Facta Univ. Ser. Math. Inform. 2 (1987), 49-52.

7. __ Problems, Aequationes Math. 39 (1990), 304.

Department of Mathematics, University of La Verne, P.O. Box 51105, Kifissia, Athens, GREECE 14510

Department of Mathematics, University of Ljubljana, Jadranska 19, 61000 LuUbluana, SLOVENIA 